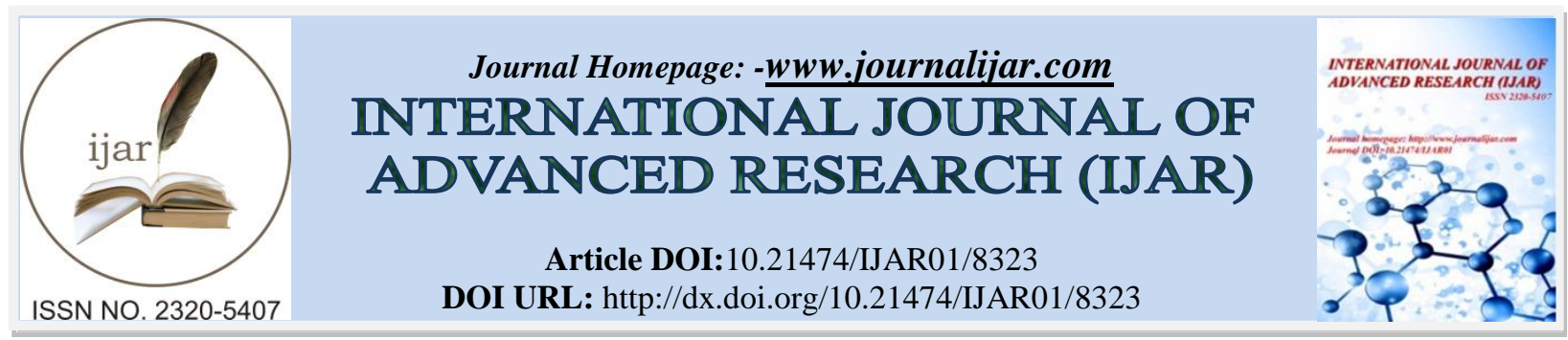

RESEARCH ARTICLE

\title{
INCREASED SPERM DNA FRAGMENTATION NEGATIVELY AFFECTS EMBRYO QUALITY IN A COHORT OF EGYPTIAN PATIENTS.
}

\author{
Gaafar $\mathrm{Sh}^{1}$, El-Geziry $\mathrm{D}^{1}$, Younan $\mathrm{D}^{\mathbf{2}}$ and El-Dakhakhny $\mathrm{M}^{\mathbf{2}}$. \\ 1. Obstetrics and Gynaecology Department, Faculty of Medicine, Alexandria University, Alexandria, Egypt. \\ 2. Clinical Pathology Department, Faculty of Medicine, Alexandria University, Alexandria, Egypt.
}

\section{Manuscript Info}

Manuscript History

Received: 04 November 2018

Final Accepted: 06 December 2018

Published: January 2019

Keywords: -

Sperm, male infertility, tunel, icsi, semen processing.

\begin{abstract}
Sperms of infertile men mostly contain more DNA damage than fertile males. Sperm DNA damage might have a negative effect on the fertility potential of such patients. This study measured the percentage of spermatozoa with nuclear DNA fragmentation in semen samples used for intracytoplasmic sperm injection (ICSI), before and after sperm processing and investigated the association between sperm DNA fragmentation index (DFI) and ICSI outcomes (embryo quality and clinical pregnancy rate).Sperm DFI was measured, by TUNEL, in fresh semen samples obtained from thirty male partners of infertile couples, prepared for ICSI.The mean sperm DFI before and after processing was $4.5 \%$ and $8.83 \%$ respectively. Sperm DFI increased significantly after processing $(\mathrm{P}<0.001)$ regardless of the type of processing used $(\mathrm{Z}=$ $0.155, p=0.877)$. The correlation between sperm DFI, before and after semen processing, and clinical pregnancy was statistically insignificant ( $\mathrm{Z}=0.4, \mathrm{p}=0.689$ and $\mathrm{Z}=0.48, \mathrm{p}=0.631$ respectively). DFI of sperm after semen processing showed a significant negative correlation with embryo grading at day $3(r=-0.232, p=0.002)$. In conclusion, sperm DFI increases significantly after processing and correlates negatively with embryo quality.
\end{abstract}

Copy Right, IJAR, 2017,. All rights reserved.

\section{Introduction: -}

Male infertility is a spectrum of abnormalities in semen parameters including; oligozoospermia, asthenozoospermia, teratozoospermia and azoospermia leading to delayed pregnancy in their female partners. It is not a single clinical entity but usually results from multiple and usually unknown etiologies. (Poongothaiet al., 2009).

There is no single definition for DNA integrity, but it can be defined as the absence of single stranded or double stranded breaks and absence of nucleotide modifications in the DNA. There are multiple levels for the loss of DNA integrity which may occur from the transformation of the spermatogonial germ cells to the ejaculated sperm. Moreover, DNA damage may affect testicular sperms, epididymal sperms or ejaculated sperms. The worst type of DNAdamage is that occurring during sperm transit and storage in the epididymis or post ejaculation as it is unrepairable. The latter could be explained by the fact that post spermiogenesis there is negligible transcription and translation (Kim, 2018).

Corresponding Author: -GaafarSh.

Address: - Obstetrics and Gynaecology Department, Faculty of Medicine, Alexandria University, Alexandria, Egypt. 
There are multiple mechanisms to explain DNA fragmentation as the abortive apoptosis theory which is due to testicular apoptosis. There is also the defective maturation theory which occurs during chromatin compaction, especially during replacement of histones by protamines. Moreover, at the post-testicular level, high oxidative stress is the most probable cause for increased DNA fragmentation and DNA base oxidation (Steger et al., 2011)

Semen processing during assisted reproductive techniques (ART) may add a further stress on sperms. Semen processing usually involves multiple centrifugation steps which may increase the oxidative damage to the sperms. So, in these cases there is another in vitro mechanism for DNA damage in addition to the previously mentioned in vivo mechanisms (Venkateshet al., 2011).

One of the technical problems in measuring DNA fragmentation is the lack of standardization of the method of evaluation. Available techniques include; sperm chromatin structure assay (SCSA), single-cell gel electrophoresis assay (COMET) acridine orange test, sperm chromatin dispersion test (Halosperm), in situ nick translation assay, and terminal deoxynucleotidyltransferase (TdT) mediated deoxyuridine triphosphate (dUTP) nick-end labelling (TUNEL) (Steger et al., 2011).

TUNEL technique is one of the most common methods which include direct quantification of sperm DNA breaks, by incorporating-flourescin labeled- dUTP at single-stranded and double stranded DNA breaks in a reaction catalyzed by the enzyme TdT. The DNA breaks, based on the incorporated dUTP, is measured by using bright field or fluorescent microscopy and flowcytometry. This is followed by classifying sperms into TUNEL positive and TUNEL negative sperms. Finally, the results are expressed as a percentage of the total sperm (Ribas-Maynou et al., 2013)

In this study, we evaluated the percentage of spermatozoa with nuclear DNA fragmentation, before and after semen processing for ICSI and investigated its association with ICSI outcome as regards embryo quality and clinical pregnancy rate.

\section{Materials and Methods: - \\ Study population: -}

This study was performed on 30 semen samples, before and after processing from 30 male partners of infertile couples undergoing ICSI trial at El-Shatby hospital, Alexandria University, between February and September2016. Couples, aged (30-50 years, mean \pm SD: $37.47 \pm 5.06$ years) of non-azoospermic men, whose female partners were younger than 35 years old (mean \pm SD: $31.5 \pm 4.39$ years) were included. All of them were suffering from primary infertility for at least 3 years (mean \pm SD: $7.03 \pm 3.71$ years) and were indicated for ICSI. Subjects with autoimmune diseases (e.g. type I diabetes mellitus), history of chemotherapy and or radiotherapy were excluded (to avoid known causes of DNA damage).

Full history was taken from all participants; including smoking habits, drug and medical history. All subjects enrolled in this study signed a written informed consent before participation. Details that might disclose the identity of the subjects under study were omitted. The study received approval of the Medical Ethics Committee of the Faculty of Medicine, Alexandria University and the practical work has been carried out in accordance with the code of Ethics of the World Medical Association (1964 Declaration of Helsinki and its later amendments).

\section{Semen Collection and Analysis: -}

Routine semen analysis was performed for all male partners participating in the present study. Semen specimens were obtained by masturbation after 3-5 days abstinence. After liquefaction, manual semen analysis was performed according to World Health Organization (WHO) 2010 guidelines (WHO, 2010). Seminal volume was determined using a graduated tube. Sperm concentration was assessed using Improved Neubauer counting chamberand expressed in millions/ml. Sperm motility was assessed in at least 200 spermatozoa within 1 hour from liquefaction and expressed as percent of total motile sperm. Sperm viability was assessed by Eosin stain, particularly in samples with poor motility. Approximately 200 spermatozoa per slide were assessed for the percentage of normal and abnormal morphology. 


\section{Sperm Processing: -}

Semen preparation was done by either the swim up technique or density gradient centrifugation, depending on the initial semen quality. Normal semen samples were subjected to the swim up technique, while poor quality samples were subjected to density gradient centrifugation.

In the swim up technique, $1 \mathrm{ml}$ of semen was placed in a sterile $15-\mathrm{ml}$ conical centrifuge tube, and $1.2 \mathrm{ml}$ of supplemented medium was gently layered over it. The tube was inclined at an angle of about $45^{\circ}$, to increase the surface area of the semen-culture medium interface, and incubated for 1 hour at $37^{\circ} \mathrm{C}$. Then, the uppermost $1 \mathrm{ml}$ of medium, which contained highly motile sperm cells, was removed. This was diluted with 1.5-2 ml of supplemented medium.The sample was centrifuged at $300 \mathrm{~g}$ for 5 minutes and the supernatant was discarded.The sperm pellet was re-suspended in $0.5 \mathrm{ml}$ of supplemented medium for assessment of sperm concentration, progressive and total motilities.

Regarding density gradient centrifugation, the density-gradient medium was prepared in a test-tube by layering $1 \mathrm{ml}$ of $40 \%(\mathrm{v} / \mathrm{v})$ density-gradient medium over $1 \mathrm{ml}$ of $80 \%(\mathrm{v} / \mathrm{v})$ density-gradient medium (SpermGrad ${ }^{\mathrm{TM}}$ Vitrolife, Sweden, ref 10102). SpermGrad ${ }^{\mathrm{TM}}$ is a bicarbonate and HEPES buffered medium containing silane-coated, colloid silica particles. The semen sample was mixed well. One $\mathrm{ml}$ of semen was placed above the density-gradient media and centrifuged at $300 \mathrm{~g}$ for 20 minutes. The supernatant was removed, and the sperm pellet was re-suspended in 5 $\mathrm{ml}$ of supplemented medium to aid removal of contaminating density-gradient medium, and then finally centrifuged at $300 \mathrm{~g}$ for 5 minutes. The washing procedure was repeated. The final pellet was re-suspended in supplemented medium (Global TotalTM LifeGlobal, Canada, product number H5GT-030) by gentle pipetting (Lópezet al., 2013).

Sperm concentration, progressive and total motilities were assessed post processing. For counting spermatozoa, improved Neubauer haemocytometer, used with special thick cover- slips, was loaded with the processed sample and assessed within 10 minutes to avoid evaporation. The concentration of spermatozoa was calculated per ml. Sperm count were assessed in both chambers of the haemocytometerat $\times 400$ magnification. Moreover, slides were examined to assess the motility of all spermatozoa within a defined area of the field at $\times 400$ magnification. Two hundred spermatozoa were being scored in two stages; progressive (PR) first, followed by non-progressive (NP) and immotile (IM) from the same area. Progressively motile spermatozoa are those moving actively, either linearly or in a large circle, regardless of speed. Non-progressive motility includes all other patterns of sperm motility with an absence of progression, e.g. swimming in small circles, the flagellar force hardly displacing the head, or when only a flagellar beat can be observed. Immotility means no sperm movement at all. Total motility includes both progressive and non-progressive motilities. The average percentage of each motility grade was calculated and approximated to the nearest whole number, i.e. PR, NP and IM. Only intact spermatozoa, defined as having a head and a tail, were assessed, i.e., motile pinheads were not counted (WHO, 2010). Then the specimen was used directly for ICSI (Lópezet al., 2013).

Assessment of Sperm DNA Fragmentationby TUNEL (Caglaret al, 2007): -

Sperm DNA fragmentation was detected in 30 semen samples before and after processing (i.e. 60 samples) by the In-SituCell Death Detection Kit, with Fluorescein label, (Roche Diagnostics, version 16.0, Cat. No. 11684795910, $\mathrm{GmbH}$, Mannheim, Germany), following the manufacturer's recommendations. This technique is based on the labeling of DNA strand breaks. Briefly, after smearing spermatozoa on slides, at a concentration that avoids their overlapping, they were air- dried and fixed with a freshly prepared fixation solution (4\% paraformaldehyde in PBS, $\mathrm{pH}$ 7.4) for $1 \mathrm{~h}$ at $15-25^{\circ} \mathrm{C}$. The slides were subsequently washed in PBS (phosphate buffer saline) and incubated in freshly preparedpermeabilisation solution $\left(0.1 \%\right.$ Triton $\mathrm{X}-100$ in $0.1 \%$ sodium citrate) for 2 min on ice $\left(2-8{ }^{\circ} \mathrm{C}\right)$. Slides were, then, rinsed twice with PBS before being incubated with FITC- labeled terminal deoxyribonucleotidyl transferase (TdT). The slides were, finally, covered and incubated in a humidified atmosphere for $60 \mathrm{~min}$ at $37^{\circ} \mathrm{C}$ in the dark. After labelling, slides were rinsed twice in PBS.

For standardization of the assay, controls were included in every experiment; for the negative control, the TdT was omitted from the reaction mix (i.e. $50 \mu \mathrm{l}$ of label solution only were added). The positive controls were prepared by incubating the sperm cells for 20 minutes at room temperature with 50 units/ml DNAse I (Boehringer Mannheim, Mannheim, Germany) prior to incubation with the TUNEL reagents.

In each slide, the total number of spermatozoa and the percentage of cells with fragmented DNA were determined by analysing each microscope field using both light and fluorescence microscopy. Stained cells were quantified on 
Olympus BX51TRF fluorescence microscope, with an excitation wavelength in the range of $450-500 \mathrm{~nm}$ and detection in the range of $515-565 \mathrm{~nm}$ (green fluorescence). A minimum of 300 spermatozoa per slide were assessed.

The percentage of spermatozoa with fragmented DNA was calculated as the number of TUNEL positive green fluorescing nuclei (FITC-labeled) in relation to the total number of sperm nuclei counted and the result was referred to as DNA fragmentation index or DFI-TUNEL (\%).

Intracytoplasmic sperm injection, Embryo Grading (Stylianouet al., 2012) and Transfer: -

After sperm injection into metaphase II oocytes, embryos were graded into 3 categories according to a) the number of blastomeres, b) evenly sized blastomeres, and c) the percentage of fragmentation intoA (good quality embryos), B (moderate quality) and $\mathbf{C}$ (poor quality). Embryos were, finally, transferred on day 3.

\section{Clinical Pregnancy: -}

Beta hCG testing was done to all female partners 2 weeks after embryo transfer (ET) and ultrasound detection of fetal sac(s) and pulsations was performed 28 days after ET.

\section{Statistical Analysis of the Data (Binuet al., 2014): -}

Data were fed to the computer and analyzed using IBM SPSS software package version 20.0. Kruskal Wallis test was used to study the relationship between \% sperm DFI in processed semenandembryo quality on day 3 , while Mann Whitney test was used to compare between any two independent variables. Wilcoxon signed ranks test was used to compare between semen parameters pre and post-processing. Spearman coefficient was used to get the correlation between day 3 embryo quality and the percentage of sperm DFI in processed semen. Significance of the obtained results was judged at the $5 \%$ level.

\section{Results: -}

The study was conducted on 30 semen samples. On raw semen, there was no statistically significant correlation between percentage of DFI and age $(\mathrm{r}=-0.119, \mathrm{p}=0.53)$ nor the duration of infertility $(\mathrm{r}=-0.113, \mathrm{p}=0.552)$. Moreover, there was no statistically significant correlation between sperm count $(\mathrm{r}=-0.126, \mathrm{p}=0.506)$ or morphology $(\mathrm{r}=-0.035, \mathrm{p}=0.853)$ and sperm DFI. However, DFI of raw semen was significantly higher in samples with lower motility $(\mathrm{r}=-0.516, \mathrm{p}=0.003)$.

Semen samples were processed by either the swim-up technique $(n=14)$ or by density gradient $(n=16)$, depending on the sample quality. Comparing semen parameters before and after processing, it was found that sperm count, motility and morphology improved after processing. However, semen processing increased mean sperm DFI significantlyfrom $4.5 \%$ to $8.83 \%(\mathrm{p}<0.001)$. Both swim-up and density gradient processing led to comparable increase in DFI $(\mathrm{Z}=0.155, \mathrm{P}=0.877)$.

To investigate the possible association between sperm DFI and ICSI outcome, embryo quality and clinical pregnancy rates were assessed. There was a significant negative correlation between the percentage of DFI of processed semen and the quality of day 3 embryos $(r=-0.232, p=0.002)$ as shown on (Table III). Moreover, samples with lower DFI resulted in significantly more class A embryos.

The overall clinical pregnancy was $50 \%(n=15)$. There was no statistically significant correlation between sperm DFI, before or after semen processing, and clinical pregnancy $(Z=0.4, p=0.689$ and $Z=0.48, p=0.631$ respectively) (Table IV).

Table 1: - Effect of semen processing on sperm DNA fragmentation index (\%)

\begin{tabular}{|l|c|c|c|}
\hline & Before Processing & After Processing & p \\
\hline DFI (\%) & $3.0(0.0-30.0)$ & $6.0(1.0-33.0)$ & $<0.001^{*}$ \\
\hline
\end{tabular}

Abnormally quantitative data expressed asmedian (min. - max.) and was compared using Wilcoxon signed ranks test

Statistically significant at $\mathrm{p} \leq 0.05$ 
Table 2: - Effect of semen processing on sperm count, motility and morphology

\begin{tabular}{|l|c|c|c|}
\hline & Raw semen & Processed semen & p \\
\hline Count (million/ml) & $39.0(7.0-126.0)$ & $41.0(10.0-150.0)$ & $<0.001^{*}$ \\
\hline Total motility (\%) & $70.0(26.0-95.0)$ & $72.50(25.0-100.0)$ & $0.001^{*}$ \\
\hline Morphology (normal, \%) & $7.0(2.0-8.5)$ & $8.0(3.5-9.5)$ & $<0.001^{*}$ \\
\hline
\end{tabular}

Data expressed as median (min. - max.) and was compared using Wilcoxon signed ranks test

Statistically significant at $\mathrm{p} \leq 0.05$

Table 3: - Relationship between sperm DNA fragmentation index (\%) and embryo quality 3 days post- processing.

\begin{tabular}{|c|c|c|c|c|}
\hline & \multicolumn{3}{|c|}{ Embryo Quality on Day 3} & \multirow[t]{2}{*}{$\mathbf{p}$} \\
\hline & $\begin{array}{l}\text { Good (A) } \\
(n=133)\end{array}$ & $\begin{array}{l}\text { Moderate (B) } \\
(\mathbf{n}=\mathbf{3 6})\end{array}$ & $\begin{array}{c}\text { Poor }(\mathbf{C}) \\
(\mathbf{n}=9)\end{array}$ & \\
\hline \% Sperm DFI in processed Semen & $3.0(1.0-25.0)$ & $6.0(1.0-33.0)$ & $6.0(3.0-20.0)$ & $<0.001^{*}$ \\
\hline Significance between groups & \multicolumn{3}{|c|}{$\frac{0.0(1.0-55.0)}{A-B^{*}}$} & \\
\hline $\mathbf{r}_{\mathrm{s}}(\mathbf{p})$ & & $-0.232^{*}\left(0.002^{*}\right)$ & & \\
\hline
\end{tabular}

Data expressed asmedian (min. - max.) and was compared using Kruskal Wallis test.

Significance between groupswas done using Mann Whitney test

$\mathrm{r}_{\mathrm{s}}$ : Spearman coefficient

*: Statistically significant at $\mathrm{p} \leq 0.05$

Grade (A) embryos: good quality embryos

Grade (B) embryos: moderate quality embryos

Grade (C) embryos: poor quality embryos

Table 4:- Correlation between sperm DNA fragmentation index $(\%)$ and clinical pregnancy after intracytoplasmic sperm injection from processed semen.

\begin{tabular}{|l|c|c|c|}
\hline \multirow{2}{*}{} & \multicolumn{2}{|c|}{ Clinical Pregnancy } & \multirow{2}{*}{$\mathbf{p}$} \\
\cline { 2 - 4 } & $\begin{array}{c}\text { Negative } \\
(\mathbf{n = 1 5})\end{array}$ & $\begin{array}{c}\text { Positive } \\
(\mathbf{n = 1 5})\end{array}$ & 0.689 \\
\hline Sperm DFI Pre-processing (\%) & $3.0(0.0-30.0)$ & $3.0(1.0-8.0)$ & 0.631 \\
\hline Sperm DFI Post-processing (\%) & $6.0(1.0-33.0)$ & $6.0(1.0-20.0)$ & \\
\hline
\end{tabular}

Data expressed asmedian (min. - max.) and was compared using Mann Whitney test

*: Statistically significant at $\mathrm{p} \leq 0.05$

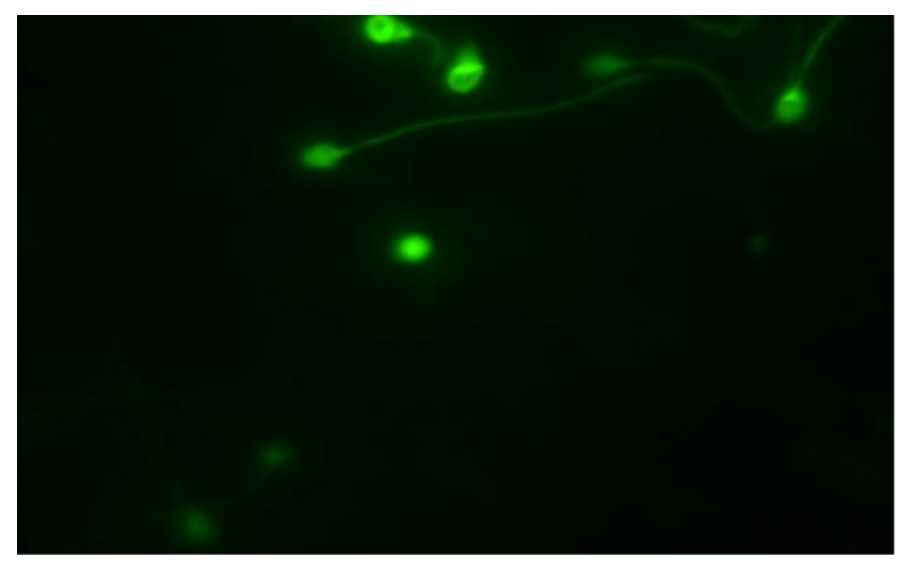

Fig 1: - Sperm cells examined for DNA damage by TUNEL assay.

The upper sperm cells are TUNEL positive (green fluorescence) while thelower 2 sperm cells are TUNEL negative under fluorescent microscope. 


\section{Discussion: -}

There have been concerns about potential consequences of the use of DNA damaged sperm in ICSI, as this technique overrides the process of natural selection. Although there has been a significant amount of research on human sperm DNA integrity over the last decade, our understanding of the mechanisms of sperm DNA damage and their effects on reproductive outcomes is still deficient (Kim, 2018).

The aim of this work was to evaluate the percentage of spermatozoa with nuclear DNA fragmentation, measured by TUNEL, in semen samples used for ICSI, before and after sperm processing. We also aimed at investigating the possible association between sperm DNA fragmentation index (DFI) and ICSI outcome (embryo cleavage and clinical pregnancy). Moreover, we examined the relationship between the percentage of sperm DFI and sperm characteristics. In addition, the effect of semen preparation on the integrity of sperm DNA and parameters of semen analysis were evaluated.

Sperm processing procedures could trigger increased production of reactive oxygen species (ROS) which begin to accumulate in the samples, and so, prolonged exposure of spermatozoa to ROS during processing, is mostly the cause of increased DFI post-processing.

There are several techniques to measure sperm DNA fragmentation and we chose the TUNEL method. A metaanalysis by Simon et al (Simon et al, 2017) of 120 studies, concluded that TUNEL and alkaline comet tests, which directly measure DNA damage, are more accurate than other methods which use indirect techniques as SCSA.

The effect of increased DFI on various ICSI outcomes was studied by various investigators. Lopez et al. 2013, could not find a significant effect of DFI on fertilization, cleavage and pregnancy rates. In their study they only included the first ICSI cycles with ejaculated sperm of 152 couples. They excluded poor responders, severe male factors, and thawed sperms from their study.

A recent study investigated the effect of increased DNA fragmentation on human embryo morphokinetics (Esbertet al., 2018). The study was done on almost 1000 embryos from 135 consecutive ICSI cycles and DNA fragmentation was measured using TUNEL. Cases with high DNA fragmentation (> 20.15\%) had significantly slower cell division than those with low fragmentation. However, DNA fragmentation did not affect embryo quality nor blastocyst formation rate.

In our studied group sperm DNA fragmentation did not have a significant effect on clinical pregnancy rate. A metaanalysis (Zhang et al 2015) also found the same conclusion that increased DNA fragmentation does not have a negative impact on pregnancy rates in ICSI cycles.

Marchesi et al. on 2010 studied the effect of semen processing technique on sperm DNA integrity using Toluidine Blue assay. The study was done on 32 semen samples. Each sample was divided and was processed by density gradient centrifugation and a modified swim-up technique. The Toluidine Blue Assay was used to analyze raw and processed samples. Both density gradient centrifugation and the modified swim-up techniques improved DNA quality compared to the unprocessed sample. However, the modified swim-up technique proved superior (i.e. the swim-up technique generated a sperm sample with better DNA integrity). These results are in contrary to our study as we found increased DNA fragmentation after processing as the mean sperm DFI before and after processing was $4.5 \%$ and $8.83 \%$ respectively. Sperm DFI increased significantly after processing $(\mathrm{P}<0.001)$ regardless the type of processing used $(\mathrm{Z}=0.155, \mathrm{p}=0.877)$. This can be explained by the type of centrifuge used, as well as the rate of centrifugation which usually varies between ART laboratories.

Panner Selvam et al on 2018 studied the redox potentials in different sperm washing media and culture media and found that oxidation-reduction potential values of the sequential culture medium and one-step culture medium were lower and significantly different $(\mathrm{p}<0.05)$ from the sperm washing media. This information can be used for selection of sperm washing media specially in those sample with high DNA fragmentation.

Among the main limitations of our study are the relatively small sample size, and the inability to follow up our female patients throughout their pregnancy to determine the miscarriage rate or live births rate in order to correlate it with sperm DFI. 
From the findings of our study, we recommend re-evaluation of the current sperm processing techniques and addition of antioxidants to media used in processing to minimize sperm DNA damage and the potential transmission of genetic mutations in assisted reproductive cycles. Moreover, developing a simplified novel DNA integrity test for the sperm to be inseminated is crucial.

\section{Conclusion: -}

In raw semen, DFI is significantly increased in samples with lower motility. In addition, semen processing significantly increases DFI. Moreover, high sperm DFI has a negative impact on quality of day 3 embryo without compromising clinical pregnancy rate.

\section{Acknowledgement: -}

The authors thank Prof. Dr. Hassan Ali El-Maghraby, head of Obstetrics and Gynecology Department, Faculty of Medicine, Alexandria University, for his support and assistance in providing the cases.

\section{References: -}

1. Binu VS, Mayya SS, Dhar M. (2014). Some basic aspects of statistical methods and sample size determination in health science research. Ayu; 35(2):119-23.

2. Caglar GS, Köster F, Schöpper B, et al. (2007). Semen DNA Fragmentation Index, Evaluated with Both TUNEL and Comet Assay, and the ICSI Outcome. in vivo; 21: 1075-1080.

3. Esbert M, Pacheco A, Soares SR, Amorós D, Florensa M et al (2018). High sperm DNA fragmentation delays human embryo kinetics when oocytes from young and healthy donors are microinjected. Androl: 12551.

4. Kim GY. (2018) What should be done for men with sperm DNA fragmentation? Clin Exp Reprod Med.;45(3):101-109

5. López G, Lafuente R, Checa MA, Carreras R, Brassesco M. (2013). Diagnostic value of sperm DNA fragmentation and sperm high-magnification for predicting outcome of assisted reproduction treatment. Asian $\mathrm{J}$ Androl; 15(6):790-4.

6. Marchesi DE, Biederman H, Ferrara S, Hershlag A, Feng HL. (2010). The effect of semen processing on sperm DNA integrity: comparison of two techniques using the novel Toluidine Blue Assay. Eur $J$ ObstetGynecolReprod Biol; 151(2):176-80.

7. Panner Selvam MK, Henkel R, Sharma R, Agarwal A. (2018). Calibration of redox potential in sperm wash media and evaluation of oxidation-reduction potential values in various assisted reproductive technology culture media using MiOXSYS system. Andrology.6(2):293-300.

8. Poongothai J, Gopenath TS, Manonayaki S. (2009). Genetics of human male infertility. Singapore Med J; 50(4):336-47.

9. Ribas-Maynou J, García-Peiró A, Fernández-Encinas A et al.(2013).Comprehensive analysis of sperm DNA fragmentation by five different assays: TUNEL assay, SCSA, SCD test and alkaline and neutral Comet assay. Andrology; 1(5):715-22.

10. Simon L, Emery BR, Carrell DT (2017). Review: Diagnosis and impact of sperm DNA alterations in assisted reproduction.Best Pract Res Clin Obstet Gynaecol.; 44:38-56.

11. Steger K, Cavalcanti MC, Schuppe HC. (2011). Prognostic markers for competent human spermatozoa: fertilizing capacity and contribution to the embryo. Int J Androl; 34:513-27.

12. Stylianou C, Critchlow D, Brison DRet al. (2012). Embryo morphology as a predictor of IVF success: an evaluation of the proposed UK ACE grading scheme for cleavage stage embryos. Hum Fertil (Camb);15(1):117.

13. Venkatesh S, Shamsi MB, Dudeja S, et al. (2011). Reactive oxygen species measurement in neat and washed semen: comparative analysis and its significance in male infertility assessment. Arch GynecolObstet; 283(1):121-6.

14. World Health Organisation. (2010). WHO Laboratory Manual for the Examination and Processing of Human Semen. 5th ed. Geneva: World Health Organisation.

15. Zhang Z, Zhu L, Jiang H, et al (2015) Sperm DNA fragmentation index and pregnancy outcome after IVF or ICSI: a meta-analysis. J Assist Reprod Genet.; 32(1):17-26. 